[RADIOCARBON, Vol. 14, No. 1, 1972, P. 247-271]

\title{
UPPSALA NATURAL RADIOCARBON MEASUREMENTS XI
}

\author{
INGRID U. OLSSON, MARTIN KLASSON, \\ and ABDALLA ABD-EL-MAGEED*
}

Fysiska institutionen, Uppsala universitet, Uppsala, Sweden

The following list covers some old measurements not included in previous lists and most of the samples measured at the Uppsala $\mathrm{C}^{14}$ laboratory since the last list (R., 1969, v. 11, p. 515-544); samples utilized for determining the increase of the $\mathrm{C}^{14} / \mathrm{C}^{12}$ ratio due to explosion of nuclear devices are omitted.

The technique used is mainly the same as previously described by Olsson (1958) and the pretreatment is also similar. In autumn 1970, we changed from bottles filled with saturated $\mathrm{CrO}_{3}$ in conc. $\mathrm{H}_{2} \mathrm{SO}_{4}$ to more $\mathrm{CrO}_{3}$ (a mixture of $60 \mathrm{~g} \mathrm{CrO}, 60 \mathrm{ml} \mathrm{H} \mathrm{H}_{2} \mathrm{O}$ and $100 \mathrm{ml}$ conc. $\mathrm{H}_{2} \mathrm{SO}_{4}$ dissolved in the same amount of water as the mixture), as suggested by A. Heikkinen, Finland. Peat, gyttja, other organic sediments, charcoal, wood, roots, leaves, and other plant remains are heated to ca. $100^{\circ} \mathrm{C}$ for some hours with $\mathrm{HCl}, 1$ to $2 \%$, left at least over night, washed with distilled water, transferred to $\mathrm{NaOH}, 1$ to $2 \%$, at $+80^{\circ} \mathrm{C}$ over night, washed with distilled water and finally acidified to $\mathrm{pH}<3$ before being dried. Combustion shortly follows pretreatment. Foraminifera and mollusk shells are leached with $\mathrm{HCl}$. Fractions used for shell samples are given in per cent as a mean value. Since shell fragments usually are different in size, the fraction of shell samples does not give the fraction of individual shells.

Bone samples were treated differently to test methods (Olsson et al., ms. in preparation). Each method is indicated with the sample. Treering samples were also treated differently as indicated in each description. Any other deviation from normal treatment is indicated in the description.

The reference sample is $95 \%$ of the $\mathrm{C}^{14} / \mathrm{C}^{12}$ ratio of the NBS oxalic-acid standard. Any corrections for apparent water ages are thus not included here, but are discussed in papers dealing with the samples. Corrections for deviations from the normal $\mathrm{C}^{13} / \mathrm{C}^{12}$ ratio $(-25.0 \%$ in the PDB scale) are applied for unknown samples. Our 6 oxalic-acid samples did not show any significant difference in their $\mathrm{C}^{31} / \mathrm{C}^{12}$ ratio according to measurements made in Stockholm. W. G. Mook in Groningen, The Netherlands, has kindly measured our 4 remaining standard samples. Oxalic acid $1,4,5$, and 6 were measured as $-19.12,-19.27$, -19.24 and $-19.62 \%$, respectively. Our secondary $\mathrm{C}^{13}$ standard, oxalicacid 1, was measured by Craig (1961) and it had a $\delta \mathrm{C}^{13}$ value of $-18.97 \%$ which should be corrected by ca. $1 \%$, giving a value of ca. $-19.2 \%$, consistent with recent measurements made by Mook for us. Since the value given by Craig in 1961 for our oxalic acid standard agrees with

* On leave from Univ. of Assuit, Assuit, Egypt. 
the international oxalic acid standard, we have not applied corrections, based on the $\mathrm{C}^{13}$ content, to our standard.

The value $5570 \mathrm{yr}$ has been used for the half-life of $\mathrm{C}^{14}$, except for some samples of geochemical interest, when $5730 \mathrm{yr}$ was used as stated in the table. These $\Delta$ values will not be changed if results are calculated with the half-life $5570 \mathrm{yr}$.

Results in this list are given B.P. (before 1950) or as an excess, $\Delta$, over the reference sample:

$$
\Delta=\delta \mathrm{C}^{14}-\left(2 \delta \mathrm{C}^{13}+50\right)\left(1+\frac{\delta \mathrm{C}^{14}}{1000}\right)
$$

where $\delta \mathrm{C}^{14}$ is the age-corrected $\mathrm{C}^{14}$ deviation from the reference sample in per mil in A.D. 1950, and $\delta \mathrm{C}^{13}$ is the deviation from PDB standard in per mil. Errors include standard deviation $(\sigma)$ of counted particles and errors in corrections due to the $\mathrm{C}^{13} / \mathrm{C}^{12}$ ratio, filling pressure, temperature, working voltage, barometric pressure, etc., as described by Olsson $(1965,1966)$. When measured activity is lower than zero, $2 \sigma$ has been used for calculation of minimum age. When it is between zero and $2 \sigma$, net activity is increased by $2 \sigma$ for calculation of minimum age. Since results are physical measurements, no terms are included for the error in the half-life or previous $\mathrm{C}^{14} / \mathrm{C}^{12}$ variations. These errors are discussed elsewhere.

Several samples had to be diluted with $\mathrm{CO}_{2}$ from an old source to bring them to normal working pressure of the counters. This has been about $2600 \mathrm{~mm} \mathrm{Hg}$ for samples with numbers U-704 or lower, proportional counter 1; ca. $1000 \mathrm{~mm} \mathrm{Hg}$ for samples with numbers from U-705 to U-757, proportional counter 1 ; and ca. $1600 \mathrm{~mm} \mathrm{Hg}$ for samples with numbers from U-2000, proportional counter 4.

\section{ACKNOWLEDGMENTS}

Description of samples are based on information provided by those who collected and submitted them. Sincere thanks are due the contributors who helped in the preparation of the text. Special thanks are also due $\mathrm{R}$. Ryhage and his co-workers for the $\mathrm{C}^{13} / \mathrm{C}^{12}$ determinations; K. Siegbahn, who has made it possible to work at the institute; and Statens Naturvetenskapliga Forkningsråd, for financial support. The authors are indebted to Carina Ericsson, Barbro Ahlström, Birgitta Wallin, and Carina Sellin for their laboratory work and to the following students who assisted: Berit Fridh, Sten-Olof Svensson, and Jan Engholm. One of us (A. A.-E.-M.) acknowledges a fellowship from the Swedish International Development Authority through the International Seminar for Research and Education in Physics.

\section{SAMPLE DESCRIPTIONS}

I. GEOLOGIC SAMPLES

A. Deep-sea cores

Indian Ocean Series 1

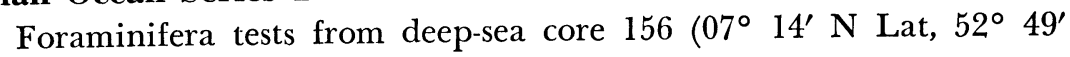


E Long), Somali Basin, Indian Ocean, depth $5107 \mathrm{~m}$. Coll. 1948 by Swedish Albatross Expedition (Pettersson); subm. by Eric Olausson, Maringeol. Lab., Univ. Göteborg, Sweden. Core is rich in coccoliths except in a few horizons (Olausson et al., 1971). One horizon dated previously U-677: $39,500+5400$ and U-678: $>3300$,500 (R., 1969, v. 11, p. 516-517). Comment: sample ultrasonically washed in acidified, boiled, distilled water, sieved, $\mathrm{pH}$ ca. 4, and dried before subm. to Uppsala.

U-2143. Core 156, w

$15,000 \pm 1000$ 13,100 в.c.

$\delta C^{13}=-1.3 \%$

Core 156, depth 170.5 to $178.5 \mathrm{~cm}, \mathrm{Pt}$. I, $>65 \mu$, whole fraction. Comment: diluted.

$$
\begin{gathered}
5300 \quad-3700 \\
3300 \text { в.с. }
\end{gathered}
$$

U.2144. Core 156, b

Core 156 , depth 170.5 to $178.5 \mathrm{~cm}$, Pt. II, $>65 \mu$, innermost $30 \%$. Comment: diluted.

U-2145. Core 156, a

Core 156 , depth 170.5 to $178.5 \mathrm{~cm}, \mathrm{Pt}$ II $>65 \mu$ out

\section{1,300 в.c.}

$\delta C^{13}=-0.3 \%$ Comment: diluted.

\section{Indian Ocean Series 2}

Foraminifera tests from deep-sea Core V16-66 (42 $39^{\prime} \mathrm{S}$ Lat, $45^{\circ}$ $40^{\prime}$ E Long), Indian Ocean, depth $3072 \mathrm{~m}$. Samples date appearance of Globorotalia truncatulinoides. Coll. 1966 by Lamont Vema Expedition; subm. by Olausson. All samples give an activity $0.022 \pm 0.036$ (weighed mean-value) corresponding to an age $>34,300$. Comment: samples ultrasonically washed in acidified, boiled distilled water, sieved, $\mathrm{pH}$ ca. 4, and dried before subm. to Uppsala. All samples diluted.

$$
\begin{array}{rr}
\text { U-2139. Vema 16-66, b } & \begin{array}{r}
>\mathbf{1 3 , 4 0 0} \\
\delta C^{13}=-4.1 \%
\end{array} \\
\text { Core V16-66, depth } 182 \mathrm{~cm},>65 \mu . \text { Innermost } 30 \% \text { was used. }
\end{array}
$$

U.2140. Vema 16-66, a

Same as U-2139; outermost $70 \%$ was used.

$$
\begin{aligned}
& >\mathbf{2 0 , 5 0 0} \\
\delta C^{1 s} & =-0.1 \%
\end{aligned}
$$

U-2141. Vema 16-66, b

$$
\begin{array}{r}
>\mathbf{3 1 , 7 0 0} \\
\delta C^{13}=-1.2 \%
\end{array}
$$

Core V16-66, depth $182 \mathrm{~cm}, 44$ to $65 \mu$. Innermost $85 \%$ was used. 


\section{U-2142. Vema 16-66, a}

Same as U-2141; outermost $15 \%$ was used.

$$
\delta C^{13}=-3.4 \%
$$

U-2135. Vema 16-66, b

$$
\begin{array}{r}
>\mathbf{2 6}, \mathbf{5 0 0} \\
\delta C^{13}=-1.8 \% \text {. }
\end{array}
$$

Core V16-66, depth $202 \mathrm{~cm},>65 \mu$. Innermost $55 \%$ was used.

U-2136. Vema 16-66, a

$$
\begin{array}{r}
>\mathbf{2 2 , 8 0 0} \\
\delta C^{13}=-2.1 \% \text { o }
\end{array}
$$

Same as U-2135; outermost $45 \%$ was used.

U-2137. Vema 16-66, b

$$
\begin{array}{r}
>\mathbf{2 9 , 0 0 0} \\
\delta C^{13}=-2.9 \% \text { o }
\end{array}
$$

Core V16-66, depth $202 \mathrm{~cm}, 44$ to $65 \mu$. Innermost $90 \%$ was used.

U-2138. Vema 16-66, a

$$
>12,800
$$

Same as U-2137; outermost $10 \%$ was used.

\section{Pacific Ocean core series}

Foraminifera from Core MSN $138 \mathrm{G}\left(00^{\circ} 15^{\prime} \mathrm{S}\right.$ Lat, $147^{\circ} 34^{\prime} \mathrm{W}$ Long). Coll. 1961 by the Monsoon Expedition. Samples were separated into fractions by sieving by acidified boiled distilled water ( $\mathrm{pH} 4)$ and leached in $\mathrm{HCl}$ to give different fractions for studies of contamination such as was seen in cores from Mediterranean (Eriksson and Olsson, 1963; Olsson and Eriksson, 1965; Olsson et al., 1968). Upper samples in core dated previously (R., 1969, v. 11, p. 517-518). Results presented in Paris, 1969 (Olsson and Eriksson, 1971). Pretreated and subm. by K. Gösta Eriksson, Inst. Geol., CTH and Univ. Gothenburg, Sweden.

$$
\begin{aligned}
& +1800 \\
& 16,600
\end{aligned}
$$

Core MSN $138 \mathrm{G}$, depth 30 to $40 \mathrm{~cm},>63 \mu$. Comment: innermost $20 \%$ was used. Diluted.

U-2149. MSN 138 G, a

$$
\begin{gathered}
\mathbf{1 8 , 0 3 0} \\
{ }^{+620} \\
\mathbf{1 6 8 0} \\
\delta C^{13}=+1.0 \% \text { B.C. }
\end{gathered}
$$

Shell layer surrounding U-2148. Comment: corresponds to outermost $80 \%$ of tests. Diluted. 


$$
\begin{gathered}
20,670 \\
\quad-470 \\
\text { 18,720 B.C. } \\
\delta C^{13}=-0.7 \% \text {. }
\end{gathered}
$$

U-2150. MSN 138 G, b

Core MSN $138 \mathrm{G}$, depth 30 to $40 \mathrm{~cm}, 4$ to $45 \mu$. Comment: innermost $35 \%$ was used.

$$
\begin{aligned}
& +840 \\
& -760
\end{aligned}
$$

Shell layer surrounding U-2150. Comment: corresponds to outermost $15 \%$ of tests. Diluted.

\section{U-2152. MSN 138 G, b}

$18,500 \pm 500$

16,550 в.C. $\delta C^{13}=+0.2 \%$

Core MSN $138 \mathrm{G}$, depth 40 to $50 \mathrm{~cm},>63_{\mu}$. Comment: innermost $45 \%$ was used. Diluted.

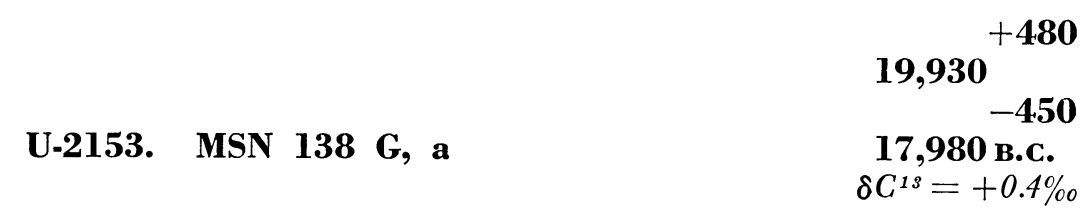

Shell layer surrounding U-2152. Comment: corresponds to outermost $55 \%$ of tests.

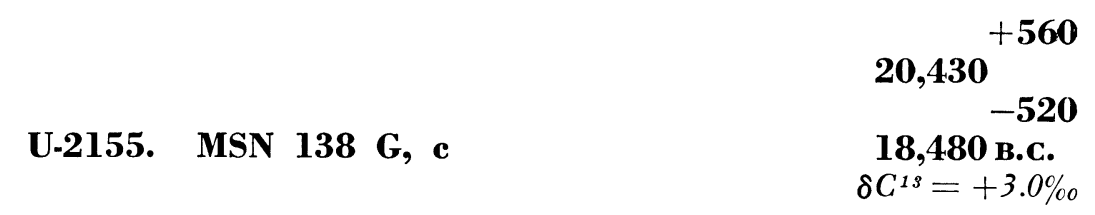

Core MSN $138 \mathrm{G}$, depth 40 to $50 \mathrm{~cm}, 4$ to $45 \mu$. Comment: innermost $50 \%$ was used.

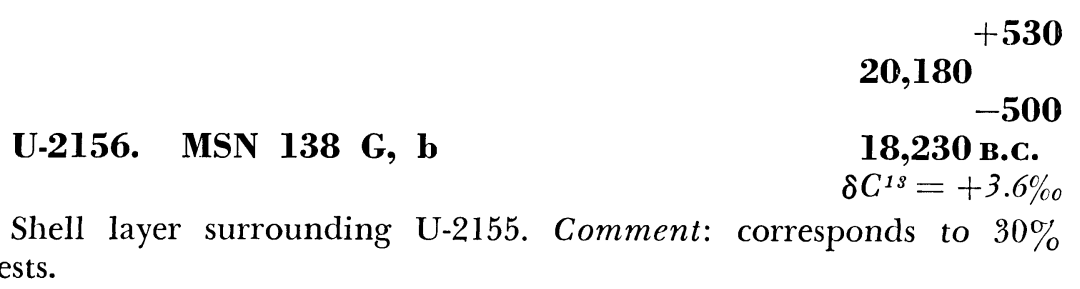
of tests. 


\section{U-2157. MSN 138 G, a}

$$
\begin{aligned}
& +540 \\
& 19,100 \\
& -510 \\
& 17,150 \text { в.C. } \\
& \delta C^{13}=-1.7 \%
\end{aligned}
$$

Shell layer surrounding U-2156. Comment: corresponds to outermost $20 \%$ of tests.

$$
\begin{gathered}
\mathbf{2 4 , 2 0 0}+\mathbf{1 1 7 0} \\
-\mathbf{1 0 2 0} \\
\mathbf{2 2 , 2 5 0 \text { в.с. }} \\
\delta C^{13}=+1.0 \% \text { o }
\end{gathered}
$$

U-2158. MSN 138 G, b

Core MSN $138 \mathrm{G}$, depth 50 to $60 \mathrm{~cm},>63 \mu$. Comment: innermost $40 \%$ was used. Diluted.

U-2159. MSN 138 G, a

$$
\begin{gathered}
\mathbf{2 4 , 0 0 0}+\mathbf{1 0 0 0} \\
-\mathbf{9 0 0} \\
\mathbf{2 2 , 1 0 0 \text { B.C. }} \\
\delta C^{13}=+0.6 \% \text { a }
\end{gathered}
$$

Shell layer surrounding U-2158. Comment: corresponds to outermost $60 \%$ of tests. Diluted.

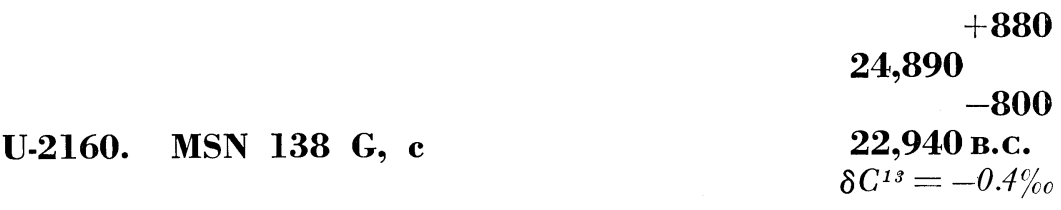

Core MSN $138 \mathrm{G}$, depth 50 to $60 \mathrm{~cm}, 4$ to $45 \mu$. Comment: innermost $35 \%$ was used.

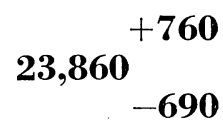

\section{U.2161. MSN 138 G, b} of tests.

Shell layer surrounding U-2160. Comment: corresponds to $45 \%$

$$
\text { U-2162. MSN } 138 \text { G, a } \begin{gathered}
\mathbf{2 3 , 1 0 0} \\
-\mathbf{8 0 0} \\
\mathbf{2 1 , 1 0 0 \mathrm { B.C }} \\
\delta C^{13}=+10.0 \%
\end{gathered}
$$

Shell layer surrounding U-2161. Comment: corresponds to outermost $20 \%$ of tests. 
$6010 \pm 190$

U-2146. Surtsey 200668, b

"Innermost" $50 \%$ was used. Comment: diluted.

4060 в.C.

$\delta C^{13}=+0.7 \%$ o

$\mathbf{1 0 , 5 7 0}$

U-2147. Surtsey 200668, a

8620 B.c.

$\delta C^{13}=-1.6 \%$

Shell layer surrounding U-2146 and smallest shell. Comment: corresponds to $35 \%$ of shells; $15 \%$ removed by washing. Diluted.

\section{Shoreline series}

Shells and peat from Iceland to determine chronology of late- and Post-glacial shorelines. Subm. by Thorleifur Einarsson, Sci. Inst., Univ. Iceland.

$\begin{array}{ll}\text { U-2225. Röndin, Kópasker, 1-70, b } & \begin{array}{l}12,830 \pm 170 \\ 10,880 \\ \end{array} \\ \delta C^{13}=+2.7 \%\end{array}$

Mya truncata from cliff Röndin at Kópasker $\left(66^{\circ} 17.5^{\prime} \mathrm{N}\right.$ Lat, $18^{\circ}$ $27^{\prime}$ W Long), Iceland, from silt layer with boulders and shells above pillow lava. Sample underlying $2 \mathrm{~m}$ moraine overlain by $1 \mathrm{~m}$ loose sand. Innermost $35 \%$ was used. Comment: difference between U-2225 and U-2226 indicates contamination. Coll. 1969 by Einarsson.

\section{U-2226. Röndin, Kópasker, 1-70, a}

$11,710 \pm 210$

9760 в.c.

$\delta C^{13}=+2.7 \%$

Shell layer surrounding U-2225. Comment: corresponds to $35 \%$ of shells; $30 \%$ removed by washing.

\section{U.724. Arnes, Vídidalur, 2-70, b}

$9730 \pm 160$

7780 B.c.

$\delta C^{13}=-2.2 \%$

Fragments of Mytilus edulis, Mya truncata, and Saxicava arctica from Árnes, Vídidalur (65 $27^{\circ} \mathrm{N}$ Lat, $20^{\circ} 36^{\prime} \mathrm{W}$ Long), Iceland, from $\mathrm{E}$ bank of $\mathrm{R}$. Vídidalsa, $15 \mathrm{~m}$ alt, in silt layer, $4 \mathrm{~m}$ thick, overlain by l m soil. Innermost $95 \%$ was used. Coll. 1969 by Einarsson.$$
9800^{+2600}
$$$$
-2000
$$$$
\text { U-725. Arnes, Vídidalur, 2-70, a } 7850 \text { в.c. }
$$$$
\delta C^{1 s}=+1.2 \% \text { o }
$$

Shell layer surrounding U-724. Comment: corresponds to $5 \%$ of shells. Whole sample only 8 g. Diluted.
} 


\section{U-2227. Kaldárbrú, 3-70, b}

Fragments of Saxicava arctica and Mya truncata from Kaldárbrú (64 $47^{\prime} \mathrm{N}$ Lat, $22^{\circ} 17^{\prime} \mathrm{W}$ Long), Kolbeinsstadahreppur, Iceland, from $5 \mathrm{~m}$ silt below $2 \mathrm{~m}$ layer with gravel, on $\mathrm{N}$ bank of R. Kaldá, near bridge. Innermost $80 \%$ was used.

$$
\begin{aligned}
& +540 \\
& \text { 11,330 }
\end{aligned}
$$

Shell layer surrounding U-2227. Comment: corresponds to $15 \%$ of shells; $5 \%$ removed by washing. Whole sample only ca. $14 \mathrm{~g}$. Diluted.

\section{U-2224. Faxaflói-bay, 2-69, INS}

Peat from floor of Faxaflói-bay ( $64^{\circ} 13.5^{\prime} \mathrm{N}$ Lat, $22^{\circ} 19^{\prime} \mathrm{W}$ Long), Melakriki, Iceland, underlying $3 \mathrm{~m}$ shelly sand. Depth $40 \mathrm{~m}$. No Betula pollen in sample; probably redeposited. Coll. 1968 by Ludvik Jonsson on $M / s$ Sandey and subm. by Einarsson.

\section{U-750. Faxaflói-bay, 2-69, SOL}

Humus products extracted from U-2224.

\section{U-2167. Tjarnargata $4,1.69$}

$9120 \pm 180$

7170 B.C.

$\delta C^{13}=-29.6 \%$

$$
1190 \pm 90
$$

A.D. 760

$\delta C^{13}=-27.2 \%$

Wood (Betula nana) from Tjarnargata 4, Reykjavik (64 $09^{\prime} \mathrm{N}$ Lat, $21^{\circ} 57^{\prime} \mathrm{W}$ Long), Iceland in peat below ground water level, with wood fragments of Larix decidua dated as U-2082: $1140 \pm 70$ (R., 1969, v. 11, p. 520-521) and bones. Coll. 1944 by Finnur Gudmundsson, Mus. Nat. Hist., Reykjavik, Iceland and subm. by Einarsson. Species determined by E. Åberg, Uppsala.

\section{Vestspitsbergen series}

C. Svalbard

Shells and whale bones from Vestspitsbergen coll. for determination of land uplift but also used for testing different methods for pretreatment of bones. Alt. given above mean sea level. Previous determinations in this series in R.: 1960, v. 2, p. 115-116; 1961, v. 3, p. 82-83; 1964, v. 6, p. $296-298$; 1965 , v. 7 , p. $317-318$; 1967, v. 9, p. $456-457$; 1969, v. 11, p. 521-524. Quaternary geology and land uplift is discussed by Birkenmajer (1960), Feyling-Hanssen (1955), Feyling-Hanssen and Olsson (19591960), and Birkenmajer and Olsson (1970). Samples from Hornsund coll. 1966 and subm. by Krzysztof Birkenmajer, Polish Acad. Sci., Krakow, 
Poland and those from Isfjorden coll. 1960 by D. H. Maling, Rolf Feyling-Hanssen, Dept. Geol., Aarhus Univ., Denmark, and I. U. Olsson. Comment: bone treatment with EDTA described in R., 1967, v. 9, p. 456.

\section{U-2229. Rettkvalbogen E, 1st, Q5a, W}

$$
\begin{aligned}
& 910 \pm 110 \\
& \text { A.D. } 1040 \\
& \delta C^{18}=-20.7 \% \text { \% }
\end{aligned}
$$

Wrong fraction from EDTA-treatment of U-2048.
$9480 \pm 180$
U-2228. Isbjørnhamna, $2 \mathbf{a}_{2}$, Q3a, Balanus, $\mathbf{b} \quad \mathbf{7 5 3 0}$ в.c.
$\delta C^{13}$ assumed $+2.8 \%$

Fragments (Balanus) from Isbj $\phi$ rnhamna $\left(77^{\circ} 00^{\prime} \mathrm{N}\right.$ Lat, $15^{\circ} 33^{\prime}$ $30^{\prime \prime}$ E Long), Wilczekodden, Hornsund, Spitsbergen, alt $7.5 \mathrm{~m}$. Sample overlay sand and gravel but underlay sand, Horizon "d" of $2 \mathrm{a}_{2}$ Terrace, Saxicava-Mya Terrace. Comment: fragments (Mya truncata, Saxicava arctica) from same batch dated previously (R., 1969, v. 11, p. 522). Innermost $55 \%$ was used.

$$
\begin{aligned}
& +210 \\
& 9470 \\
& -200 \\
& \text { U-727. Isbjørnhamna, } 2 a_{2}, \text { Q3a, Balanus, a } 7520 \text { в.c. } \\
& \delta C^{13}=+2.8 \% \text { o }
\end{aligned}
$$

Shell layer surrounding U-2228. Comment: corresponds to $25 \%$ of shells; $20 \%$ removed by washing.

\section{U-2270. Kapp Linné 6005, Al, R}

Vertebra of whale from Kapp Linné ( $78^{\circ} 04^{\prime} \mathrm{N}$ Lat, $13^{\circ} 40^{\prime} \mathrm{E}$ Long), Isfjorden, Spitsbergen, alt ca. $1 \mathrm{~m}$. Coll. 1960 by Feyling-Hanssen and Olsson. Comment: sample treated with acetone before EDTA extraction. Diluted.

$$
\begin{array}{r}
\mathbf{4 6 9 0}+\mathbf{8 0 0} \\
-\mathbf{7 5 0} \\
\mathbf{2 7 4 0 \text { B.c. }} \\
\delta C^{13}=-25.4 \% 0
\end{array}
$$

U-2271. Kapp Linné 6005, Al, A

Acetone extract from U-2270. Comment: probably contaminated with oil from radio sta. Diluted.

\section{U-2272. Kapp Linné $6005, \mathrm{B1}, \mathbf{R}$}

Sample from same vertebra as U-2270. Comment: not treated with acetone before EDTA extraction. 
U-2273. Ekholmvika 6022 b, HCI, $<250 \mu$, r

Insoluble remains after $\mathrm{HCl}$ treatment of whalebone from Ekholmvika ( $78^{\circ} 35^{\prime} \mathrm{N}$ Lat, $16^{\circ} 38^{\prime} \mathrm{E}$ Long), Billefjorden, Spitsbergen; alt ca. $50 \mathrm{~m}$. Deeply buried in fine gravel. Coll. 1960 by D. H. Maling. Comment: grains $<250 \mu$ used. $\mathrm{HCl}$ added until $\mathrm{pH}$ of liquid remained at 1 to 1.5 for 4 days. $1-\mathrm{N} \mathrm{HCl}$ used.

$$
\begin{gathered}
\mathbf{7 0 1 0} \text { B.C. } \\
\delta C^{13}=-18.1 \% 0
\end{gathered}
$$

In $\mathrm{HCl}$ soluble fraction of bone received at treatment giving U-2273.

$$
\begin{aligned}
& 9660 \pm 180 \\
& \text { U-2275. Ekholmvika } 6022 \text { b, HCI, 250-500 } \mu, \mathbf{r} 7710 \text { B.c. } \\
& \delta C^{13}=-16.6 \% \text { o }
\end{aligned}
$$

Insoluble remains after $\mathrm{HCl}$ treatment of whalebone also used for U-2273. Comment: grains 250 to $500 \mu$ used; $\mathrm{pH} 1$ to 1.5 for 7 days. 1-N HCl used.

$$
\begin{array}{r}
7700 \\
-510
\end{array}
$$

U.751. Ekholmvika 6022 b, HCl, 250-500 $\mu, 1 \quad 5750$ в.C.

In $\mathrm{HCl}$ soluble fraction of bone received at treatment giving U-2275. Comment: diluted.

\section{U-169. Gipshuken 6016 a, inorganic}

$$
\begin{array}{r}
\Delta=\mathbf{3 1 2 1} \pm \mathbf{3 0} \% \\
\delta C^{13} \text { assumed }-20.0 \% \text { o }
\end{array}
$$

Inorganic fraction of bone of $\mathrm{U}-467, \mathrm{CO}_{2}$ liberated at HCl-treatment, with assumed false result. Comment: sample was pulverized before treatment. Ca. $25 \% \mathrm{HCl}$ used. Sample stored in room with high $\mathrm{C}^{14}$ content before treatment. Diluted.

\section{Sörmon series}

$$
\text { D. Sweden }
$$

Charcoal from Sörmon (59 23' N Lat, $13^{\circ} 22^{\prime}$ E Long), Värmland, Sweden. Blomquist (1969) has described Sörmon formation. Coll. 1968 and subm. by Thorsten Blomquist, Inst., Quaternary Geol., Univ. Uppsala, Sweden.

\section{U-2133. Sörmon 8C}

$7060 \pm 180$

5110 B.C.

Charcoal from Quarry VII, ca. $0.5 \mathrm{~m}$ below surface. 


\section{U-2132. Sörmon 10C}

Charcoal from Quarry VII, ca. $0.6 \mathrm{~m}$ below surface.

\section{Kyrkviken series}

Post-glacial sediments from Kyrkviken (62 $55^{\prime} \mathrm{N}$ Lat, $17^{\circ} 47^{\prime} \mathrm{E}$ Long), $1 \mathrm{~km} \mathrm{~S} \mathrm{Kramfors,} \mathrm{Ångermanland,} \mathrm{Sweden.} \mathrm{Samples} \mathrm{from} \mathrm{sharp} \mathrm{transition}$ from black clay rich in sulphur to light-gray clay with somewhat disturbed micro-varves with organic substance. Salinity analyses by Birgitta Ericsson, diatom analyses by Maj-Britt Florin, both of Inst. Quaternary Geol., Univ. Uppsala, Sweden. Brackish-water sediments. Carbon content ca. $1.4 \%$ according to Ericsson. Contamination with graphite not excluded. Kyrkviken sediments described by Ericsson (1970). Coll. 1969 and subm. by Ericsson. Comment: due to risk of contamination with allochtonous material, sediments are not suitable for dating; different fractions must be studied. $\mathrm{NaOH}$-soluble fraction dated at lower age than insoluble fraction, as seen previously in similar samples (R., 1964, v. 6, p. 303; 1969 , v. 11, p. 532). Insoluble fraction difficult to combust, in comparison with peat, gyttja, or soluble fraction. Total yield in radiocarbon lab. corresponds to $<1 \%$ carbon. A detailed description will be given by Olsson, 1971.

\section{U.713. Kyrkviken 1, INS}

$12,660 \pm 260$

Insoluble part, 0 to $5 \mathrm{~cm}$ above transition.

\section{$+1200$ \\ U-714. Kyrkviken 1, SOL

In warm dilute $\mathrm{NaOH}$ soluble part from U-713. Comment: diluted.

\section{U-715. Kyrkviken 2, INS}

Insoluble fraction, 0 to $5 \mathrm{~cm}$ above transition. Comment: diluted.

\section{U-716. Kyrkviken 2, INS + SOL, 1st}

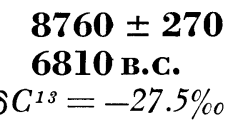

In warm dilute $\mathrm{NaOH}$ soluble part mixed with insoluble part from U-715. Fraction corresponds to gas received at 1st step of combustion, when sample is heated very little. Comment: diluted. 


\section{U-717. Kyrkviken 2, INS + SOL, 2nd}

14,200 в.c.

$\delta C^{13}=-27.9 \%$

Fraction corresponds to gas received at 2nd step of combustion of U-716. Sample is heated more and stronger stream of oxygen is used. Comment: diluted.

\section{U-718. Kyrkviken 3, INS}

$8970 \pm 250$

7020 B.c.

$\delta C^{13}=-28.8 \%$

Insoluble part, 0 to $5 \mathrm{~cm}$ below transition. Comment: diluted.

U-719. Kyrkviken 3, SOL

In warm dilute $\mathrm{NaOH}$ soluble part from U-718. Comment: diluted.

\section{U-2276. Graphite 200169, C}

Graphite from chemicals dealer, Kebo. Comment: gas obtained at normal combustion. High temperature, strong stream of oxygen, bad yield. Diluted.

\section{Fjällsjö series}

Sediments from Sil, Fjällsjö $\left(63^{\circ} 46^{\prime} 13^{\prime \prime} \mathrm{N}\right.$ Lat, $16^{\circ} 27^{\prime} 43^{\prime \prime} \mathrm{E}$ Long), Angermanland, Sweden, alt $225 \mathrm{~m}$. Coll. 1969 and subm. by Erling Lindström, Inst. Nat. Geog., Univ. Uppsala, Sweden.

\section{U-707. Sil, Fjällsjö, I, INS}

Peat, 63 to $65 \mathrm{~cm}$ below surface.

$2650 \pm 150$

700 B.c.

$\delta C^{1 s}=-33.0 \%$ o

\section{U-2205. Sil, Fjällsjö, I, SOL}

Humus products extracted from U-707.

$2540 \pm 80$

590 B.c.

$\delta C^{13}=-27.4 \%$

\section{U-2206. Sil, Fjällsjö, II, INS}

$2660 \pm 80$

Silt-gyttja, 65 to $67 \mathrm{~cm}$ below surface. 


\section{U-2207. Sil，Fjällsjö，II，SOL}

Humus products extracted from U-2206.

U-708. Sil, Fjällsjö, III, INS

$$
\begin{gathered}
2620 \pm \mathbf{8 0} \\
\mathbf{6 7 0} \text { B.c. } \\
\delta C^{13}=-26.8 \% \circ
\end{gathered}
$$

10,920

$+350$

$-340$

8970 B.c.

$\delta C^{13}=-27.2 \%$ surface.

Silt with low content of organic material, 118 to $120 \mathrm{~cm}$ below

$$
\begin{gathered}
10,300 \\
-1500 \\
\text {-1300 } \\
8300 \text { в.C. } \\
\delta C^{13}=-29.1 \% \text { o }
\end{gathered}
$$

U-709. Sil, Fjällsjö, III, INS + SOL 8300 в.C.

Mixture of soluble and insoluble fractions from same level as U-708. Comment: ca. $2 / 3$ insoluble fraction removed. Sample corresponds to ca. 1/3 insoluble and all soluble fraction. Diluted.

$$
\begin{array}{cc} 
& +1000 \\
\text { U-710. Sil, Fjällsjö, III, SOL } & \mathbf{8 6 0 0} \\
\text { Humus products extracted from U-708. Comment: } & \mathbf{6 0 0} \\
\delta C^{13}=-28.6 \% 0 \\
\text { diluted. }
\end{array}
$$

\section{Bjurselet series}

Gyttja from Bjurselet (65 $10^{\prime} \mathrm{N}$ Lat, $21^{\circ} 10^{\prime} \mathrm{E}$ Long), Byske parish, Västerbotten, Sweden, to date vegetational development recognized in pollen diagrams related to archaeologic studies (see Archaeologic Samples, this series, below) in same area. Pollen analyses by Lars-König Königsson and Thorolf Candolin, Inst. Quaternary Geol., Univ. Uppsala, Sweden. Geol. description by Königsson (1970) including Königsson's interpretation of $\mathrm{C}^{14}$ dates. Geol. samples dated previously (R., 1967, v. 7, p. 454-470). Coll. 1964 and subm. by Königsson. Comment: all samples highly humified and roots removed by sieving before subm. to radiocarbon lab.; earlier described as carr peat, information when samples were subm., based on preliminary field studies.

\section{U-500. Bjurselet 14 G, INS}

$2600 \pm 600$

700 B.c.

Sandy silt with gyttja substance, 56 to $53.5 \mathrm{~cm}$ below surface, below big decrease of Salix. Comment: diluted. Very small remainder used to certify that $14 \mathrm{G}$ younger than $11 \mathrm{G}$. Complete new pretreatment. 


\title{
U-612. Bjurselet 11 G, INS
}

\author{
$3970 \pm 70$ \\ 2020 B.c. \\ $\delta C^{13}=-29.1 \%$ o
}

Silty gyttja, 48.5 to $46 \mathrm{~cm}$ below surface. $\mathrm{CO}_{2}$ from new combustion, after treatment with $\mathrm{HCl}$, of sample pretreated for U-579. Below 1st appearance of cerealia and Hordeum.

\section{U-600. Bjurselet 6 G, INS}

$3610 \pm 60$

1660 B.c.

$$
\delta C^{13}=-28.6 \% \text { 。 }
$$

Silty gytjja with wood fragments, 38 to $36 \mathrm{~cm}$ below surface. Before beginning of Picea.

\section{U-2056. Bjurselet 6 G, INS}

$3450 \pm 90$

1500 B.C.

Same $\mathrm{CO}_{2}$ as for $\mathrm{U}-600$, but measured in another counter.

\section{U-602. Bjurselet 4 G, INS}

$$
\begin{array}{r}
2630 \pm 80 \\
680 \text { B.c. } \\
\delta C^{13}=-29.7 \% 0
\end{array}
$$

Peaty gyttja with wood fragments, 34 to $32 \mathrm{~cm}$ below surface. Dates maximum of Pinus and minimum of Alnus.

\section{U-601. Bjurselet 3 G, INS}

$$
\begin{array}{r}
2420 \pm 80 \\
\mathbf{4 7 0} \text { в.c. } \\
\delta C^{13}=-28.8 \% \propto
\end{array}
$$

Peaty gyttja with wood fragments, 32 to $29 \mathrm{~cm}$ below surface. Dates minimum of Pinus and Picea, although both show increasing tendency in pollen curves. Also dates maximum of Alnus in curve showing decreasing tendency and decrease of Betula. Gramineae and Sphagnum sp. increase strongly.

\section{ARCHAEOLOGIC SAMPLES}

\section{Ivory coast series}

\section{A. Africa}

Shells and charcoal from shell mounds adjacent to lagoons, Ivory Coast. Samples dated to prove that shell accumulations are artificial. Mounds generally lense-shaped; formerly regarded as natural deposits, but Dorthe (1964) and Madon (1967, 1969) reported bone and pottery finds. Raymond Mauny, Fac. Lettres et Sci. Humaines, Univ. Paris, France, described sites archaeologically (1971). Present radiocarbon dates discussed by Mauny and Olsson (mss. in preparation). Some dates on different fractions of 1 sample given previously, U-264: $970 \pm 110$, U-265: $990 \pm 70$ and U-266: $950 \pm 70$ (R., 1964, v. 6, p. 294-295), from $+15 \mathrm{~m}$, not $48 \mathrm{~m}$ as Tricart stated. Comment: Pachymelania byronensis lives in slightly brackish water, Egeria paradoxa in fresh water, and Aloidis trigona in brackish water. Only one possibility given to determine apparent age of water by cross-dating with charcoal from same site. Dif- 
ference between weighed mean value of shells (U-2194 and U-2195, Aloidis) and charcoal (U-753) is $385 \pm 120$.

\section{U-2184. Songon Kassemblé, c}

$$
\begin{aligned}
& 1200 \pm 90 \\
& \text { A.D. } 750 \\
& \delta C^{13}=-6.1 \%
\end{aligned}
$$

Aloidis trigona from Songon Kassemblé $\left(05^{\circ} 18^{\prime} \mathrm{N}\right.$ Lat, $04^{\circ} 17^{\prime} \mathrm{W}$ Long), Daval, $22 \mathrm{~km} \mathrm{~W}$ Abidjan, from $1 \mathrm{~m}$ depth in mound, +0.2 to $1.65 \mathrm{~m}$. Coll. 1969 and subm. by Mauny. Comment: innermost 40\% was used.

\section{U-2185. Songon Kassemblé, b}

$$
1430 \pm 130
$$
shells.

Shell layer surrounding U-2184. Comment: corresponds to $15 \%$ of

\section{U-2186. Songon Kassemblé, a}

\section{A.D. 820}

$$
\delta C^{13}=-8.2 \%
$$

Shell layer surrounding U-2185. Comment: corresponds to $25 \%$ of shells; $20 \%$ removed by washing.

\section{U.2187. Songon Dagbé, 1b}

$$
2630 \pm 120
$$

Aloidis trigona from Songon Dagbé $\left(05^{\circ} 18^{\prime} \mathrm{N}\right.$ Lat, $04^{\circ} 18^{\prime} \mathrm{W}$ Long), $23 \mathrm{~km} \mathrm{~W}$ Abidjan, $1 \mathrm{~m}$ depth in mound, +0 to $8 \mathrm{~m}$, containing copper bracelets, axes, pottery and iron harpoon. Coll. 1969 and subm. by Mauny Comment: innermost $30 \%$ was used.

\section{U-2188. Songon Dagbé, la}

$$
\begin{gathered}
2640 \pm 130 \\
690 \text { B.C. } \\
\delta C^{13}=-11.5 \% \%
\end{gathered}
$$

Shell layer surrounding U-2187. Comment: corresponds to $35 \%$ of shells; $35 \%$ removed by washing.

\section{U-2189. Songon Dagbé, 2c}

$$
\begin{gathered}
\mathbf{2 8 4 0} \pm \mathbf{1 5 0} \\
\mathbf{8 9 0} \text { B.C. } \\
\delta C^{13}=-10.8 \% \%
\end{gathered}
$$

Aloidis trigona from same colln. as U-2187. Comment: innermost $30 \%$ was used.

\section{U-2190. Songon Dagbé, 2b}

$$
\begin{gathered}
2730 \pm 100 \\
780 \text { B.C. }
\end{gathered}
$$

$$
\delta C^{13}=-7.7 \%
$$
shells.

Shell layer surrounding U-2189. Comment: corresponds to $40 \%$ of 


\section{U-2191. Songon Dagbé, 2a}

Shell layer surrounding U-2190. Comment: corresponds to $30 \%$ of shells; $0 \%$ removed by washing.

\section{U-2192. Dabou-Tchotchoraf, -2 , b}

$2920 \pm 100$ 970 B.C.

$\delta C^{13}=-11.4 \%$

Aloidis trigona from Dabou-Tchotchoraf $\left(05^{\circ} 18^{\prime} 51^{\prime \prime} \mathrm{N}\right.$ Lat, $04^{\circ}$ $21^{\circ} 56^{\prime \prime} \mathrm{W}$ Long), $2 \mathrm{~km}$ ESE Dabou, from $2 \mathrm{~m}$ depth in mound, +1 to $5.15 \mathrm{~m}$, containing pottery. Coll. 1969 and subm. by Mauny. Comment: innermost $40 \%$ was used.

U-2193. Dabou-Tchotchoraf, -2, a

$2920 \pm 140$

970 B.c.

$\delta C^{13}=-11.8 \%$

Shell layer surrounding U-2192. Comment: corresponds to $15 \%$ of shells; $45 \%$ removed by washing.

U-2194. Dabou-Tchotchoraf, -1.1, b

$2920 \pm 80$

970 в.C.

$\delta C^{13}=-10.1 \%$

Aloidis trigona from same mound as U-2192, from $1.1 \mathrm{~m}$ depth in mound. Comment: innermost $25 \%$ was used.

\section{U-2195. Dabou-Tchotchoraf, -1.1, a}
$3010 \pm 140$
1060 в.C.
$\delta C^{13}=-10.4 \%$

Shell layer surrounding U-2194. Comment: corresponds to $40 \%$ of shells; $35 \%$ removed by washing.

\section{U-2196. Tiebissou, b}

$$
\begin{gathered}
3630 \pm 100 \\
1680 \text { в.C. } \\
\delta C^{13}=-8.5 \% \text {. }
\end{gathered}
$$

Egeria paradoxa from Tiebissou $\left(05^{\circ} 20^{\prime} \mathrm{N}\right.$ Lat, $04^{\circ} 5 \mathrm{I}^{\prime} \mathrm{W}$ Long), $100 \mathrm{~km} \mathrm{~W}$ Abidjan, on the 2 banks of river at Bandama, $23 \mathrm{~km}$ from coast. Sample from several sites 4 to $8 \mathrm{~m}$ above level of river. Coll. 1967 by H. Madon, Sodemi, Abidjan and subm. by Mauny. Comment: innermost $35 \%$ was used.

\section{U-2197. Tiebissou, a}

$3750 \pm 150$

1800 B.C.

$\delta C^{13}=-11.8 \%$

Shell layer surrounding U-2196. Comment: layer corresponds to $35 \%$ of shells; $30 \%$ removed by washing.

U-2198. Ehoussou, $-1.7, \mathrm{~b}$

$3010 \pm 100$

1060 B.c.

$\delta C^{13}=-12.1 \%$

Pachymelania byronensis from Ehoussou, near lagoon Aby $\left(05^{\circ} 14^{\prime}\right.$ $21^{\prime \prime} \mathrm{N}$ Lat, $03^{\circ} 16^{\prime} 20^{\prime \prime} \mathrm{W}$ Long), $82 \mathrm{~km} \mathrm{E}$ Abidjan, from $1.7 \mathrm{~m}$ depth 
in mound, +0.1 to $0.9 \mathrm{~m}$, containing bones. Coll. 1967 by Melseaux, Abidjan and subm. by Madon. Comment: innermost $25 \%$ was used. Sample probably contaminated by $\mathrm{CO}_{2}$ dissolved in water penetrating into bottom layers of mound.

\section{U-2199. Ehoussou, -1.7 , a}

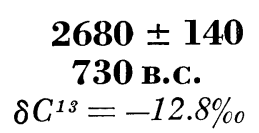

Shell layer surrounding U-2198. Comment: corresponds to $35 \%$ of shells; $40 \%$ removed by washing.

\section{U-2200. Ehoussou, $-1.0, \mathrm{~b}$}

Pachymelania byronensis from same mound as U-2198, from $1.7 \mathrm{~m}$ depth. Comment: innermost $25 \%$ was used.

U-2201. Ehoussou, -1.0 , a

$$
3210 \pm 150
$$

Shell layer surrounding U-2200. Comment: corresponds to $35 \%$ of shells; $40 \%$ removed by washing.

\section{U2202. Ehoussou, $-0.3, \mathrm{~b}$}

$3340 \pm 100$ 1390 B.C.

Pachymelania byronensis from same mound as U-2198 and U-2200, from $0.3 \mathrm{~m}$ depth. Comment: innermost $25 \%$ was used.

\section{U-2203. Ehoussou, -0.3 , a}

Shell layer surrounding U-2202. Comment: corresponds to $35 \%$ of shells; $40 \%$ removed by washing.

\section{U-753. Dabou-Tchotchoraf, $-1.1, \mathrm{k}$}

$$
\begin{gathered}
2560 \pm 90 \\
610 \text { B.c. } \\
\delta C^{13}=-25.9 \% o
\end{gathered}
$$
diluted.

Charcoal from soil separated from shells, part of U-2194. Comment:

\section{B. Sweden}

\section{Gårdlösa series}

Charcoal from Iron age settlement Gårdlösa No. 2 (grave-field) and No. 3 (house foundations) $\left(55^{\circ} 34^{\prime} \mathrm{N}\right.$ Lat, $14^{\circ} 08^{\prime} \mathrm{E}$ Long), Smedstorp parish, Skåne, Sweden. Coll. and subm. by Berta Stjernquist, Hist. Mus., Univ. Lund, Sweden. Several samples were dated previously (R., 1965, v. 7, p. 326-327; 1967; v. 9, p. 465-467; 1969, v. 11, p. 536.) 
U-746. Gårdlösa 2, Stone-settings 1967, No. $67: 8$

$$
\begin{aligned}
& \mathbf{1 5 5 0} \pm \mathbf{8 0} \\
& \text { A.D. 400 } \\
& \delta C^{13}=-26.5 \% 。
\end{aligned}
$$

Charcoal from 0.55 to $0.65 \mathrm{~m}$ below surface, with melted bronze, fragment of iron, bead, and fragment of a comb. Coll. 1967.

U-706. Gårdlösa 2, Stone-settings 1967, No. $67: 11$

$1800 \pm 80$ A.D. 150 $\delta C^{13}=-25.9 \%$

Charcoal from 0.45 to $0.60 \mathrm{~m}$ below surface, with melted bronze, fragment of an iron pin, and bead. Coll. 1967.

U-2222. Gårdlösa 2, Stone-settings 1967 No. $67: 16$

$$
1600 \pm 80
$$

A.D. 350

$\delta C^{13}=-24.5 \%$

Charcoal from 0.55 to $0.65 \mathrm{~m}$ below surface, with melted bronze, fragments of bronze sheets, iron fragments, beads, and parts of 2 spindle whirles. Coll. 1967.

\section{U-705. Gårdlösa 2, Stone-settings 1967} No. $67: 27$

$$
\begin{array}{r}
1300 \pm 70 \\
\text { A.D. } 650 \\
\delta C^{13}=-28.3 \%
\end{array}
$$

Charcoal from 0.45 to $0.55 \mathrm{~m}$ below surface, with part of the bronze spring of a fibula, iron fragments, beads, and melted glass. Coll. 1967.

U-2219. Gårdlösa 2, Stone-settings 1967, No. $67: 32$

$$
\begin{gathered}
\mathbf{1 4 3 0} \pm \mathbf{7 0} \\
\text { A.D. 520 } \\
\delta C^{13}=-25.4 \% \text { o }
\end{gathered}
$$

Charcoal from 0.58 to $0.63 \mathrm{~m}$ below surface with bones of a dog. Coll. 1967. Comment: part of sample dated previously, U-672: $1340 \pm 70$.

U-2220. Gårdlösa 2, Stone-settings 1967,

$$
\text { No. } 67: 33
$$

$$
\begin{array}{r}
\mathbf{1 5 0 5} \pm \mathbf{7 5} \\
\text { A.D. 445 } \\
\delta C^{13}=-25.5 \%
\end{array}
$$

Charcoal from 0.55 to $0.70 \mathrm{~m}$ below surface, with a bead. Coll. 1967 .

U-2221. Gårdlösa 2, Stone-settings 1967,

$$
\text { No. } 67: 39
$$

$$
\begin{gathered}
1360 \pm \mathbf{8 0} \\
\text { A.D. 590 } \\
\delta C^{13}=-24.9 \%
\end{gathered}
$$

Charcoal from 0.50 to $0.65 \mathrm{~m}$ below surface, with bronze fragment with ornament, and bead. Coll. 1967.

\section{U.747. Gårdlösa 3, House LI}

$$
\begin{aligned}
& 1310 \pm 100 \\
& \text { A.D. } 640 \\
& \delta C^{13}=-24.0 \%
\end{aligned}
$$

Charcoal from layer, underlying 20-cm-thick layer with cultivated soil from 0.65 to $1.05 \mathrm{~m}$ below surface, with potsherds and a bead. Coll. 1966. 


\section{U-2261. Gårdlösa 3, House XLVII}

$$
\begin{aligned}
& 1550 \pm \mathbf{8 0} \\
& \text { A.D. } 400 \\
& \delta C^{13}=-24.3 \% o
\end{aligned}
$$

Charcoal from layer, underlying 20-cm-thick layer with cultivated soil, from 0.70 to $0.95 \mathrm{~m}$ below surface, with fibula of bronze, iron and bronze pin, fragments of knives, bead, and potsherds. Coll. 1965.

\section{U-752. Ödehoburga $1^{21}$}

$$
\begin{gathered}
\mathbf{2 2 9 0} \pm \mathbf{1 3 0} \\
\mathbf{3 4 0} \text { B.c. } \\
\delta C^{13}=-24.3 \%
\end{gathered}
$$

Charcoal from ödehoburga $\left(57^{\circ} 54^{\prime} \mathrm{N}\right.$ Lat, $19^{\circ} 6^{\prime} \mathrm{E}$ Long), Fårö, Gotland, Sweden, from big piece of charcoal in hearth with potsherds. Coll. 1970 and subm. by Anna-Lena Gerdin, Gotland's Hist. Mus., Visby, Sweden.

\section{Bjurselet series}

Charcoal and bones from Bjurselet $\left(65^{\circ} 00^{\prime} \mathrm{N}\right.$ Lat, $21^{\circ} 04^{\prime} \mathrm{E}$ Long), Byske parish, Västerbotten, Sweden. From Stone age dwelling-site, alt $53 \mathrm{~m}$, with imported flint axes, scrapers, and potsherds. Upper layer consisted of cultivated soil, Layer I, underlain by sand, Layers II, III, and IV (Christiansson, 1965a, b, 1970). Samples dated previously (R., 1967 , v. 9 , p. $467-469 ; 1969$, v. 11, p. 539). Pollen profile from same area discussed by Königsson (1970), with his interpretation of radiocarbon dates from Uppsala lab., partly pub. previously (R., 1969, v. 9, p. 465; this list, above). Subm. by Christiansson, Inst. Nordic Antiquities, Univ. Uppsala, Sweden.

\section{U-2223. Bjurselet 116/266, Härdplatsen}

Charcoal from hearth. Coll. 1968 by Christiansson.

$$
\begin{array}{r}
\mathbf{4 8 0} \pm \mathbf{7 0} \\
\text { A.D. } 1470 \\
\delta C^{1 s}=-25.0 \%
\end{array}
$$

\section{U-2204. Bjurselet 194/230, Åkern}

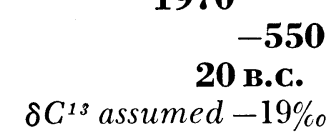

Burned seal bone (Pusa hispida) from field, Layer II and III. Coll. 1966 by Christiansson. Comment: sample EDTA-treated, but was so small that "right" and "wrong" fractions were combined and dated as one sample. Diluted.

\section{TREE-RING SAMPLES}

\section{Northern Sweden series}

Pinus from Hörnett at Hörnsjön (63 $17^{\prime} \mathrm{N}$ Lat, $18^{\circ} 401 / 2^{\prime}$ E Long), Ångermanland, Sweden. Tree from hill S of lake, $100 \mathrm{~m}$ alt, felled 1969, age $56 \mathrm{yr}$, ca. $1.5 \mathrm{~m}$ above ground. Softwood. Subm. by A. Assarsson, Mo and Domsjö AB, Örnsköldsvik, Sweden, where part of wood was 
chemically treated as described previously (R., 1969, v. 11, p. 539-540). Standardized treatment was applied on part of wood (Uppsala) as a check. General Comment: "atomic-bomb effect" seen in the extract from tree rings grown as early as A.D. 1950-1945 is assumed due to radial movement of nutrients in the parenchyma cells. Extraction with $\mathrm{NaOH}(1 \%$ b. w.) also gave an extract showing "atomic-bomb effect", although slightly smaller than that observed following more sophisticated treatment. Treatment of remaining wood at Uppsala gave higher activity than the holocellulose and wood extracted at Mo and Domsjö, implying that standard treatment with $\mathrm{NaOH}$ may be insufficient for precise work such as calibration of radiocarbon time scale. A similar result was observed for Fitzroya (see "Argentina series" below).

\begin{tabular}{|c|c|c|c|c|}
\hline \multicolumn{5}{|c|}{ Age-corrected excess in activity of Pinus wood, A.D. 1945-1950 } \\
\hline Lab. no. & Substance & $\delta \mathrm{C}^{13} \%$ & $\Delta \% \mathrm{~T} 1 / 2=5730$ & Prepared in \\
\hline U-2168 & Holocellulose & -22.8 & $-35.6 \pm 7.8$ & Örnsköldsvik \\
\hline U-2169 & Extracted wood & -23.1 & $-42.0 \pm 7.0$ & Örnsköldsvik \\
\hline $\mathrm{U}-2170$ & Extract & -27.4 & $+145.8 \pm 10.2$ & Örnsköldsvik \\
\hline U-2171 & Extracted wood (INS) & -25.1 & $-13.9 \pm 6.7$ & Uppsala \\
\hline U-2172 & Extract (SOL) & -13.6 & $+114.3 \pm 22.2$ & Uppsala \\
\hline
\end{tabular}

\section{Argentina series}

Tree trunk (Fitzroya cupressoides) from Parque Nac. Los Alerces (43 $\mathrm{S}$ Lat, $71^{\circ} \mathrm{W}$ Long), Esquel, Chubut, Argentina. Park created 1937. Trunk donated by Intendencia, Parc Nac. Los Alerces via Åke Vinterbäck, Univ. Uppsala, Sweden. Tree rings counted by Horring, High School of Forestry, Stockholm, Sweden. Tree probably was felled A.D. 1934. Cellulose and lignin prepared according to Olson and Broecker (1958), the 2 cellulose fractions, however, with slightly different procedures. One sample separated into fractions at Mo and Domsjö (see N Sweden series, above; R., 1969, v. 11, p. 539-540). Some results pub. previously (R., 1969, v. 11, p. 541). Results from same trees pub. by Lerman et al. (1970).

\begin{tabular}{lcclrrr}
\hline \multicolumn{3}{c}{ Age before } & Age B.P. & & \multicolumn{3}{c}{ Age-corrected excess } \\
Lab. no. & A.D. 1934 & $(1950)$ & Substance & $\delta \mathrm{C}^{13} \%$ o & $\delta \mathrm{C}^{14} \%$ \% & $\Delta \%$ \\
\hline U-2281 & $619-610$ & 630.5 & Lignin & -24.6 & -17.3 & $-18 \pm 9$ \\
U-2282 & $619-610$ & 630.5 & Cellulose w & -22.8 & -19.7 & $-24 \pm 9$ \\
U-2283 & $619-610$ & 630.5 & Cellulose y & -22.9 & -22.6 & $-27 \pm 9$ \\
U-2284 & $589-580$ & 600.5 & Holocellulose & -22.4 & -2.7 & $-8 \pm 5$ \\
U-2285 & $589-580$ & 600.5 & Extracted wood & -24.6 & -6.3 & $-7 \pm 5$ \\
U-743 & $589-580$ & 600.5 & Extract & -25.1 & +20.4 & $+21 \pm 8$ \\
\hline
\end{tabular}


IV. GEOCHEMICAL SAMPLES

\section{Tree leaves series}

Tree leaves coll. to investigate influence of dissolved carbonate on $\mathrm{C}^{14} / \mathrm{C}^{12}$ ratio in trees. Contemporaneous $\mathrm{C}^{14} / \mathrm{C}^{12}$ ratio of atmosphere will be given later (Olsson, ms. in preparation). Present samples from Uppland, from area with soft ground-water and those from öland, from area with hard ground-water (Olsson et al., 1969).

\section{U-689. Salix, Järlåsa 1006 68, INS

$$
\begin{array}{r}
\delta \mathbf{C}^{14}=+587 \pm \mathbf{1 1} \% o \\
\Delta=+\mathbf{6 0 0} \pm \mathbf{1 1} \% o \\
\delta C^{13}=-29.0 \% \circ
\end{array}
$$

Leaves (Salix) at ditch with water at Rönningen $\left(17^{\circ} 13^{\prime} \mathrm{N}\right.$ Lat, $59^{\circ}$ 581/2' E Long), Norra Hagunda; Uppland, Sweden. Coll. 1968 by Olsson.

\section{U-690. Betula, Järlåsa 1006 68, INS}

$$
\begin{array}{r}
\delta \mathbf{C}^{14}=+\mathbf{5 9 3} \pm \mathbf{1 1} \% \circ \\
\Delta=+\mathbf{6 0 1} \pm \mathbf{1 1} \% \circ \\
\delta C^{13}=-27.5 \% 0
\end{array}
$$

Leaves (Betula) at Hedbolund (17 $13^{\prime} \mathrm{N}$ Lat, $59^{\circ} 56^{\prime} \mathrm{E}$ Long), Norra Hagunda, Uppland, Sweden. Coll. 1968 by Olsson.

\section{U-691. Salix, MM 1006 68, INS}

$$
\begin{array}{r}
\delta \mathbf{C}^{14}=+611 \pm \mathbf{9} \% \\
\Delta=+623 \pm 9 \% \\
\delta C^{13}=-28.5 \% \circ
\end{array}
$$

Leaves (Salix) at Möckelmosen (56 $36^{\circ} \mathrm{N}$ Lat, $16^{\circ} 31^{\prime} \mathrm{E}$ Long), Öland, Sweden. Coll. approx. same day as Järlåsa samples, 1968 by Königsson.

\section{U-692. Salix, MM 1006 68, SOL}

In warm dilute $\mathrm{NaOH}$ soluble fraction of U-691.

$$
\begin{array}{r}
\delta \mathbf{C}^{14}=+\mathbf{5 7 6} \pm \mathbf{8} \% \circ \\
\Delta=+\mathbf{5 8 8} \pm \mathbf{8} \% \circ \\
\delta C^{13}=-28.8 \% \circ
\end{array}
$$

\section{U-693. Betula, MM 1006 68, INS}

$$
\begin{array}{r}
\delta \mathbf{C}^{14}=+\mathbf{5 7 8} \pm \mathbf{9} \% \\
\Delta=+\mathbf{5 9 2} \pm \mathbf{9} \% \\
\delta C^{13}=-29.5 \%
\end{array}
$$

Leaves (Betula) at Möckelmosen (56 $32^{\prime} \mathrm{N}$ Lat, $16^{\circ} 31^{\prime} \mathrm{E}$ Long), Öland, Sweden. Coll. approx. same day as Järlåsa samples, 1968 by Königsson.

\section{U-694. Betula, MM 1006 68, SOL}

$$
\begin{array}{r}
\delta \mathbf{C}^{14}=+\mathbf{5 8 5} \pm \mathbf{9} \% \circ \\
\Delta=+\mathbf{5 9 5} \pm \mathbf{9} \% \circ \\
\delta C^{13}=-28.2 \% \circ
\end{array}
$$

In warm dilute $\mathrm{NaOH}$ soluble fraction of $\mathrm{U}-693$.

\section{Plant series}

Tomato plants grown to investigate influence of dissolved carbonate on $\mathrm{C}^{14} / \mathrm{C}^{12}$ ratio in plants. Contemporaneous $\mathrm{C}^{14} / \mathrm{C}^{12}$ ratio of atmosphere will be given later (Olsson, ms., in preparation). Plants grown in plastic buckets on balcony outside $\mathrm{C}^{14}$ lab., 1968. 


\section{U-2278. Tomato 68, L, C12, INS}

$$
\begin{array}{r}
\delta \mathbf{C}^{14}=+\mathbf{5 7 5} \pm \mathbf{1 0} \\
\Delta=+\mathbf{5 7 6} \pm \mathbf{1 0} \\
\delta C^{13}=-25.2 \% 0
\end{array}
$$

Tomato leaves grown in peat to which $\mathrm{C}^{14}$-free carbonate was added.

\section{U-2279. Tomato 68, L, Cn, TW, INS}

$$
\begin{array}{r}
\delta \mathbf{C}^{14}=+\mathbf{5 9 0} \pm \mathbf{1 2} \\
\Delta=+\mathbf{5 9 0} \pm \mathbf{1 2} \\
\delta C^{13}=-25.8 \% 0
\end{array}
$$

Tomato leaves grown in peat. Watered by rain and tap water.

\section{U-2280. Tomato 68, L, C14, INS}

$$
\begin{array}{r}
\delta \mathbf{C}^{14}=+\mathbf{5 7 6} \pm \mathbf{9} \\
\Delta=+\mathbf{5 6 5} \pm \mathbf{9} \\
\delta C^{13}=-21.8 \% \circ
\end{array}
$$

Tomato leaves grown in peat to which $6 \mathrm{~g} \mathrm{CaCO}_{3}$ with $\Delta=3940$ \pm 80 was added, $\delta \mathrm{C}^{13}$ assumed $-25 . \%$.

\section{U.757. Tomato 68, L, C14, SOL}

$$
\begin{array}{r}
\delta \mathbf{C}^{14}=+\mathbf{5 6 9} \pm \mathbf{1 7} \\
\Delta=+\mathbf{5 8 5} \pm \mathbf{1 7} \\
\delta C^{13}=-30.1 \% 0
\end{array}
$$

In warm dilute $\mathrm{NaOH}$ soluble fraction extracted from U-2280.

\section{U-754. Tomato 68, R, C12, INS}

$$
\begin{array}{r}
\delta \mathbf{C}^{14}=+\mathbf{5 5 0} \pm \mathbf{1 2} \\
\Delta=+\mathbf{5 5 1} \pm \mathbf{1 2} \\
\delta C^{13}=-25.2 \%
\end{array}
$$

Roots of tomato plant used for U-2278. Comment: diluted.

\section{U-755. Tomato 68, R, Cn, TW, INS}

$$
\begin{array}{r}
\delta \mathbf{C}^{14}=+\mathbf{5 9 0} \pm \mathbf{1 1} \\
\Delta=+\mathbf{5 9 3} \pm \mathbf{1 1} \\
\delta C^{13}=-26.0 \%
\end{array}
$$

Roots of tomato plants used for U-2279.

\section{U-756. Tomato 68, R, C14, INS}

$$
\begin{array}{r}
\delta \mathbf{C}^{14}=+\mathbf{5 7 7} \pm \mathbf{1 2} \\
\Delta=+\mathbf{5 8 2} \pm \mathbf{1 2} \\
\delta C^{13}=-26.7 \% \text { o }
\end{array}
$$
diluted.

Roots of tomato plant used for U-2280 and U-757. Comment:

\section{Corrections to earlier lists}

R., 1967, v. 9, p. 457: U-506 should be U-482.

R., 1967, v. 9, p. 458: U-2019. Ekruhorn, 11,620 \pm 240 (9670 B.c.) B.C.).

R., 1967, v. 9, p. 463: U-468. Lillsjön I, 70 to $74,9180 \pm 380$ (7230

R., 1969, v. 11, p. 523: U-2121 should be U-2120. 


\section{REFERENCES}

Alexandersson, Torbjörn, 1970, The sedimentary xenoliths from Surtsey: Marine sediments lithified on the sea-floor: Surtsey Research progress rept., v. 5, p. 83-89. 1971, The sedimentary xenoliths from Surtsey: Turbidites indicating shelf growth: Surtsey Research progress repts., v. 6, in press.

Birkenmajer, Krzysztof, 1960, Raised marine features of the Hornsund area, Vestspitsbergen: Studia Geol. Polon., v. 5, 95 p.

Birkenmajer, Krzysztof and Olsson, I. U., 1971, Radiocarbon dating of raised marine terraces at Hornsund, Spitsbergen, and the problem of land uplift; Norsk Polarinst. Årb., 1969, p. 17-43.

Blomquist, Thorsten, 1969, Sörmon, stratigrafiska studier av en litoralt omlagrad glaciofluvial ackumulation i Värmland: Mimeo. paper, Inst. Quaternary Geol., Univ. Uppsala, Sweden, 33 p.

Christiansson, Hans, 1965a, De arkeologiska undersökningarna vid Bjurselet i Byske: Västerbotten, p. 191-202.

—_ 1965b, Flint finds in Västerbotten; Hunting, trade or agriculture?, in: Hunting and Fishing, Nordic symposium in Luleå 1962, Norrbottens Mus., Luleå, Sweden, p. 91-110.

1970, Archaeological introduction, in Christiansson H. (ed.): The Bjurselet settlement. A Neolithic settlement on the Byske river, Västerbotten, Northern Sweden, Sect. 1: Acta Soc. Skytteanæ, 1970, no. 7, p. 5-11.

Craig, Harmon, 1961, Mass-spectometer analyses of radiocarbon standards: Radiocarbon, v. 3, p. 1-3.

Dorthe, J. P., 1964, Reconnaissance rapide des faluns lagunaires de Côte-d'Ivoire. Leur interêt économique: Rapport SODEMI, no. 83, Abidjan, 38 p.

Ericsson, Birgitta, 1970, Salthaltsvariationer inom postglaciala lersediment från Kyrkviken, Angermanland: Mimeo. paper, Inst. Quaternary Geol., Univ. Uppsala, Sweden, $101 \mathrm{p}$.

Eriksson, K. G. and Olsson, I. U., 1963, Some problems in connection with $\mathrm{C}^{14}$ dating of tests of Foraminifera: Geol. Inst. Univ. Bull., Uppsala, v. 42, p. 1-13.

Feyling-Hanssen, R. W., 1955, Stratigraphy of the marine late-Pleistocene of Billefjorden, Vestspitsbergen: Norsk Polarinst., Skr. 107, 186 p.

Feyling-Hanssen, R. W. and Olsson, Ingrid, 1959-1960, Five radiocarbon datings of post glacial shorelines in central Spitsbergen: Norsk Geog. Tidsskr., v. 17, p. 122-131.

Königsson, L.-K., 1970, Traces of Neolithic human influence upon the landscape development at the Bjurselet settlement, Västerbotten, Northern Sweden, in: Christiansson, H. (ed.): The Bjurselet settlement. A Neolithic settlement on the Byske river, Västerbotten, Northern Sweden, Sect. 1: Acta Soc. Skytteanæ, 1970, no. 7, p. 13-30.

Lerman, J. C., Mook, W. G., and Vogel, J. C., 1970, C ${ }^{14}$ in tree rings from different localities, in: Olsson, I. U. (ed), Nobel Symposium 12. Radiocarbon variations and absolute chronology, p. 275-301.

Madon, H., 1967, Inventaire des faluns lagunaires de la partie occidentale de la lagune ébrié (campagne 1966-67): Rapport SODEMI, no. 00, Abidjan, 21 p.

1969, Premier inventaire des gîtes de sables coquillers de Côte-d'Ivoire:
Rapport SODEMI, no. 229, Abidjan, 56 p. Rapport SODEMI, no. 229, Abidjan, 56 p.

Mauny, Raymond, 1971, Contribution à la connaissance de l'archéologie préhistorique et protohistorique ivoiriennes: Inst. recherche de l'Univ. d'Abidjan Bull.

Olausson, Eric, Bilal ul Haq, U. Z., Karlsson, G. B., and Olsson, I. U., 1971, Evidence in Indian Ocean cores of Late Pleistocene changes in oceanic and atmospheric circulation: Geol. Fören. Stockholm, Förh., v. 93, p. 51-84.

Olson, E. A. and Broecker, W. S., 1958, Sample contamination and reliability of radiocarbon dates: N.Y. Acad. Sci. Trans., ser. 2, v. 20, p. 593-604.

Olsson, Ingrid, 1958, A C ${ }^{14}$ dating station using the $\mathrm{CO}_{2}$ proportional counting method: Arkiv f. Fysik, v. 13, p. 37-60.

1960, Uppsala natural radiocarbon measurements II: Am. Jour. Sci. Radiocarbon Supp., v. 2, p. 112-128.

1965, Computer calculations of $\mathrm{C}^{14}$ determinations: 6th internatl. conf. radiocarbon and tritium dating Proc., Pullman, Washington, June 7-11, 1965, p. $383-392$.

1966, Computer calculations of $\mathrm{C}^{14}$ determinations: Uppsala Univ. Inst. Physics Rep., UUIP-477, 11 p. 
Olsson, Ingrid, 1971, The pretreatment of samples and the interpretation of the results of $\mathrm{C}^{14}$ determinations, in: Symposium on climatic changes in arctic areas during the last 10,000 years, Oct. 1971, Oulu, Finland, in press.

Olsson, Ingrid, Cazeneuve, Horacio, Gustavsson, John, and Karlén, Ingvar, 1961, Uppsala natural radiocarbon measurements III: Radiocarbon, v. 3, p. 81-85.

Olsson, I. U., El-Gammal, S., and Göksu, Yeter, 1969, Uppsala natural radiocarbon measurements IX: Radiocarbon, v. 11, p. 515-544.

Olsson, I. U. and Eriksson, K. G., 1965, Remarks on $\mathrm{C}^{14}$ dating of shell material in sea sediments: Prog. in Oceanog., v. 3, p. 253-266.

1969, in press.
19actionation studies of shells of foraminifera: INQUA Proc., Paris

Olsson, I. U., Göksu, Yeter, and Stenberg, Allan, 1968, Further investigation of storing and treatment of foraminifera and mollusks for $\mathbf{C}^{14}$-dating: Geol. Fören. Stockholm, Förh., v. 90, p. 417-426.

Olsson, I. U. and Kilicci, Serap, 1964, Uppsala natural radiocarbon measurements IV: Radiocarbon, v. 6, p. 291-307.

Olsson, I. U. and Piyanuj, Piya, 1965, Uppsala natural radiocarbon measurements V: Radiocarbon, v. 7, p. 315-330.

Olsson, I. U., Stenberg, Allan, and Göksu, Yeter, 1967, Uppsala natural radiocarbon measurements VII: Radiocarbon, v. 9, p. 454-470. 